\title{
ON LATTICE-TOPOLOGICAL PROPERTIES OF GENERAL WALLMAN SPACES
}

\author{
CARMEN VLAD \\ Mathematics Department \\ Pace University \\ Pace Plaza, New York, NY 10038 U.S.A.
}

(Received November 30, 1995 and in revised form March 25, 1996)

\begin{abstract}
Let $X$ be an arbitrary set and $\mathcal{L}$ a lattice of subsets of $X$ such that $\emptyset, X \in \mathcal{L} . \mathcal{A}(\mathcal{L})$ is the algebra generated by $\mathcal{L}$ and $I(\mathcal{L})$ consists of all zero-one valued finitely additive measures on $\mathcal{A}(\mathcal{L})$ Various subsets of $I(\mathcal{L})$ are considered and certain lattices are investigated as well as the topology of closed sets generated by them. The lattices are investigated for normality, regularity, repleteness and completeness. The topologies are similarly discussed for various properties such as $T_{2}$ and Lindelof
\end{abstract}

KEY WORDS AND PHRASES: Normal, Lindelöf, countable compact lattices; strongly $\sigma$-smooth measures

1991 AMS SUBJECT CLASSIFICATION CODES: 28C15, $28 \mathrm{~A} 12$.

\section{INTRODUCTION}

Let $X$ be an arbitrary set and $\mathcal{L}$ a lattice of subsets of $X$ such that $\emptyset, X \in \mathcal{L} . \quad \mathcal{A}(\mathcal{L})$ is the algebra generated by $\mathcal{L}$ and $I(\mathcal{L})$ denotes those non trivial, zero-one valued, finitely additive measures on $\mathcal{A}(\mathcal{L})$

Various subsets of $I(\mathcal{L})$ are considered and certain lattices in these subsets are investigated as well as the topology of closed sets generated by them. The lattices are investigated for normality, regularity, and for a variety of repleteness and completeness conditions. The topologies are similarly investigated for various properties such as $T_{2}$ and Lindelöf. Necessary and sufficient conditions for these properties to hold can effectively be given in terms of measure conditions on the original lattice.

Some investigations in these matters have been begun in [2], [3] and [8]. We go beyond these results, and introduce new subsets of $I(\mathcal{L})$ and their lattices to investigate.

We begin with some standard notations and terminology that will be used throughout the paper Our notations and terminology is consistent with that in the literature (see e.g. [1],[2],[5] and [10]), and is added mainly for the reader's convenience.

We then proceed in the subsequent sections to analyze in detail the lattice-topological structure of various Wallman spaces as indicated above.

\section{BACKGROUND AND NOTATIONS}

Let $X$ be an arbitrary nonempty set, and $\mathcal{L}$ a lattice of subsets of $X$. It is assumed throughout the paper that $\emptyset, X \in \mathcal{L}$.

We adhere to the customary lattice-topological definitions which can be found for example in [1],[2],[4],[7] and [10]. Here, we just note some of the measure theoretic equivalents. For this purpose we introduce the following notations: $\mathcal{A}(\mathcal{L})$ denotes the algebra generated by $\mathcal{L}$, and $I(\mathcal{L})$ the set of 
non-trivial zero-one valued finitely additive measures on $\mathcal{A}(\mathcal{L}) \quad I_{R}(\mathcal{L})$ the set of $\mathcal{L}$-regular measures of $I(\mathcal{L})$, where $\mu \in I(\mathcal{L})$ is $\mathcal{L}$-regular if for any $A \in \mathcal{A}(\mathcal{L}) \mu(A)=\sup \{\mu(L) / L \subset A, L \in L\}$ sequences $\left\{L_{n}\right\}$ of sets of $\mathcal{L}$ with $L_{n} \downarrow \phi, \mu\left(L_{n}\right) \rightarrow 0 . I^{\sigma}(\mathcal{L})$ the set of $\sigma$-smooth measures of $I(\mathcal{L})$ on $\mathcal{L}$, where $\mu \in I(\mathcal{L})$ is $\sigma$-smooth on $\mathcal{L}$ if for all of $\sigma$-smooth measures on $\mathcal{A}(\mathcal{L})$ of $I(\mathcal{L}) I_{R}^{o}(\mathcal{L})$ the set of $\mathcal{L}$-regular measures of $I^{\sigma}(\mathcal{L}) . \quad \pi(\mathcal{L})=\{\Pi$, defined on $\mathcal{L}$, non-trivial, monotone, and $\Pi(A \cap B)=\Pi(A) \Pi(B), A, B \in \mathcal{L}\}$ the set of all premeasures on $\mathcal{L} \pi_{\sigma}(\mathcal{L})$ is the set of all premeasures on $\mathcal{L}$ which are $\sigma$-smooth on $\mathcal{L}$

Note that there exists a one-to-one correspondence between

$\mathcal{L}$-filters $\mathcal{F}$ and elements of $\pi(\mathcal{L})$ given by $\Pi(L)=1$ iff $L \in \mathcal{F}$.

$\mathcal{L}$-filters with countable intersection property and $\pi_{\sigma}(\mathcal{L})$.

All elements of $I(\mathcal{L})$ and all prime $\mathcal{L}$-filters, given by:

for any $\mu \in I(\mathcal{L})$ we associate the prime $\mathcal{L}$-filter given by

$$
\mathcal{F}=\{A \in \mathcal{L} / \mu(A)=1\} .
$$

All elements of $I_{R}(\mathcal{L})$ and all $\mathcal{L}$-ultrafilters, given by the following rule. with each $\mathcal{L}$-ultrafilter $\mathcal{F}$ we associate the zero-one valued measure defined on $\mathcal{A}(\mathcal{L})$ by:

$$
\mu(E)=\left\{\begin{array}{l}
1 \text { if there exists } A \in \mathcal{F}, A \subset E \\
0 \text { if there exists } A \in \mathcal{F}, A \subset E^{\prime} .
\end{array} .\right.
$$

The support of $\mu \in I(\mathcal{L})$ is $S(\mu)=\cap\{L \in \mathcal{L} / \mu(L)=1\}$. With this notation, we now note $\mathcal{L}$ is compact iff $S(\mu) \neq \emptyset$ for every $\mu \in I_{R}(\mathcal{L}) . \quad \mathcal{L}$ is countably compact iff $I_{R}(\mathcal{L})=I_{R}^{o}(\mathcal{L}) . \quad \mathcal{L}$ is normal iff for each $\mu \in I(\mathcal{L})$, there exists a unique $\nu \in I_{R}(\mathcal{L})$ such that $\mu \leq \nu(\mathcal{L})$ i.e. $\mu(L) \leq \nu(L)$ for all $L \in \mathcal{L}$ $\mathcal{L}$ is regular iff whenever $\mu_{1}, \mu_{2} \in I(\mathcal{L})$ and $\mu_{1} \leq \mu_{2}(\mathcal{L})$, then $S\left(\mu_{1}\right)=S\left(\mu_{2}\right) . \mathcal{L}$ is replete iff for any $\mu \in I_{R}^{o}(\mathcal{L}), S(\mu) \neq \emptyset . \quad \mathcal{L}$ is prime-complete iff for any $\mu \in I_{\sigma}(\mathcal{L}), S(\mu) \neq \emptyset . \quad \mathcal{L}$ is Lindelof iff for any $\Pi \in \pi_{\sigma}(\mathcal{L}), S(\Pi) \neq \emptyset$. Finally, if $\mu_{x}$ is the measure concentrated at $x \in X$ then $\mu_{x} \in I_{R}(\mathcal{L})$, for all $x \in X$, iff $\mathcal{L}$ is disjunctive.

For further results and related matters see [6], [8] and [9].

\section{THE SPACES $I_{R}^{\sigma}(\mathcal{L}), I_{\sigma}(\mathcal{L})$ AND THE LATTICES $\mathcal{W}_{\sigma}(\mathcal{L}), \mathcal{V}_{\sigma}(\mathcal{L})$}

We consider in this section the important space $I_{R}^{\sigma}(\mathcal{L})$; for $A \in \mathcal{A}(\mathcal{L})$ define

$$
W_{\sigma}(A)=\left\{\mu \in I_{R}^{\sigma}(\mathcal{L}) \mid \mu(A)=1\right\} .
$$

Then, assuming $\mathcal{L}$ is disjunctive, $\mathcal{W}_{\sigma}(\mathcal{L})=\left\{W_{\sigma}(L) / L \in \mathcal{L}\right\}$ is a lattice in $I_{R}^{\sigma}(\mathcal{L})$ isomorphic to $\mathcal{L}$, under the map $L \rightarrow W_{\sigma}(L), L \in \mathcal{L}$, and $\mathcal{A}\left(\mathcal{W}_{\sigma}(\mathcal{L})\right)=\mathcal{W}_{\sigma}(\mathcal{A}(\mathcal{L}))$. Also, the map $\mu \rightarrow \mu^{\prime}$, where $\mu^{\prime}\left(W_{\sigma}(A)\right)=\mu(A), A \in \mathcal{A}(\mathcal{L})$ is a bijection between $I_{R}^{\sigma}(\mathcal{L})$ and $I_{R}^{\sigma}\left(\mathcal{W}_{\sigma}(\mathcal{L})\right)$. It is well known that $\mathcal{W}_{\sigma}(\mathcal{L})$ is replete and is a basis for the closed sets $\tau \mathcal{W}_{\sigma}(\mathcal{L})$, all arbitrary intersections of sets of $\mathcal{W}_{\sigma}(\mathcal{L})$ It is this topological space $I_{R}^{\sigma}(\mathcal{L}), \tau \mathcal{W}_{\sigma}(\mathcal{L})$, and lattice $\mathcal{W}_{\sigma}(\mathcal{L})$ which we will consider here and subsequent sections. Analogously, we also consider $I_{\sigma}(\mathcal{L})$ and $\mathcal{V}_{\sigma}(\mathcal{L})$; here we do not need the assumption of disjunctiveness on $\mathcal{L}$, and $\mathcal{V}_{\sigma}(\mathcal{L})=\left\{V_{\sigma}(L) / L \in \mathcal{L}\right\}$ where $V_{\sigma}(A)=\left\{\mu \in I_{\sigma}(\mathcal{L}) / \mu(A)=1\right\}, A \in \mathcal{A}(\mathcal{L})$. $\mathcal{V}_{\sigma}(\mathcal{L})$ is prime complete, and is a base for the closed sets $\tau \mathcal{V}_{\sigma}(\mathcal{L})$ of $I_{\sigma}(\mathcal{L})$. A few of the properties to be considered have been investigated in [3], we give slightly different proofs, and include some of them for completeness.

THEOREM 3.1 a) Consider $I_{R}^{\sigma}(\mathcal{L})$ and $\mathcal{W}_{\sigma}(\mathcal{L})$ with $\mathcal{L}$ disjunctive. $\mathcal{W}_{\sigma}(\mathcal{L})$ is regular iff for all $\mu_{1}, \mu_{2} \in I(\mathcal{L})$ and $\nu \in I_{R}^{\sigma}(\mathcal{L})$, if $\mu_{1} \leq \mu_{2}(\mathcal{L})$ and $\mu_{1} \leq \nu(\mathcal{L})$ then $\mu_{2} \leq \nu(\mathcal{L})$

b) The topological space $I_{R}^{\sigma}(\mathcal{L}), \tau \mathcal{W}_{\sigma}(\mathcal{L})$ with $\mathcal{L}$ disjunctive is considered then the space is $T_{2}$ iff for $\mu \in I(\mathcal{L})$ and $\mu \leq \nu_{1}(\mathcal{L}), \mu \leq \nu_{2}(\mathcal{L})$ where $\nu_{1}, \nu_{2} \in I_{R}^{\sigma}(\mathcal{L})$ it follows that $\nu_{1}=\nu_{2}$.

c) Consider $I_{\sigma}(\mathcal{L})$ and $\mathcal{V}_{\sigma}(\mathcal{L}) . \quad \mathcal{V}_{\sigma}(\mathcal{L})$ is regular iff for all $\mu_{1}, \mu_{2} \in I(\mathcal{L})$ and $\nu \in I_{\sigma}(\mathcal{L})$ if $\mu_{1} \leq \mu_{2}(\mathcal{L})$ and $\mu_{1} \leq \nu(\mathcal{L})$ then $\mu_{2} \leq \nu(\mathcal{L})$. 
d) Consider the topological space $I_{\sigma}(\mathcal{L}), \tau \mathcal{V}_{\sigma}(\mathcal{L})$. This space is $T_{2}$ iff for $\mu \in I(\mathcal{L})$ with $\mu \leq \nu_{1}(\mathcal{L})$ and $\mu \leq \nu_{2}(\mathcal{L})$ where $\nu_{1}, \nu_{2} \in I_{\sigma}(\mathcal{L})$, it follows $\nu_{1}=\nu_{2}$

PROOF. The proofs for a) and c) and for b) and d) are similar. We just prove a) and b)

a) Let $\mu_{1}, \mu_{2} \in I(\mathcal{L})$ such that $\mu_{1} \leq \mu_{2}(\mathcal{L})$ Then there exist $\mu_{1}^{\prime}, \mu_{2}^{\prime} \in I\left(\mathcal{W}_{o}(\mathcal{L})\right)$ and $\mu_{1}^{\prime}\left(W_{\sigma}(L)\right)=\mu_{1}(L), \mu_{2}^{\prime}\left(W_{\sigma}(L)\right)=\mu_{2}(L)$ for all $L \in \mathcal{L} . \quad \mu_{1}(L) \leq \mu_{2}(L) \Rightarrow \mu_{1}^{\prime} \leq \mu_{2}^{\prime}$ on $\mathcal{W}_{\sigma}(\mathcal{L})$ Suppose $\mathcal{W}_{\sigma}(\mathcal{L})$ is regular Then $S\left(\mu_{1}^{\prime}\right)=S\left(\mu_{2}^{\prime}\right)$ where

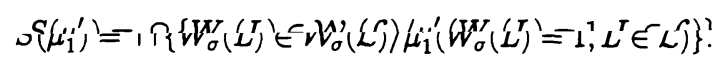

Let now $\nu \in I_{R}^{\sigma}(\mathcal{L})$ with $\mu_{1} \leq \nu$. We have $\nu^{\prime} \in I_{R}^{\sigma}(\mathcal{W}(\mathcal{L}))$ and $\mu_{1}^{\prime} \leq \nu^{\prime}$ on $\mathcal{W}_{\sigma}(\mathcal{L})$, therefore $S\left(\nu^{\prime}\right) \subset S\left(\mu_{1}^{\prime}\right)=S\left(\mu_{2}^{\prime}\right)$; hence $\mu_{2}^{\prime} \leq \nu^{\prime}$ on $\mathcal{W}_{\sigma}(\mathcal{L})$ i.e. $\mu_{2} \leq \nu$ on $\mathcal{L}$. Conversely, let $\mu_{1}, \mu_{2} \in I(\mathcal{L})$ and $\nu \in I_{R}^{\sigma}(\mathcal{L})$ such that if $\mu_{1} \leq \mu_{2}(\mathcal{L})$ and $\mu_{1} \leq \nu(\mathcal{L})$ then $\mu_{2} \leq \nu(\mathcal{L})$. Let now $\lambda_{1}, \lambda_{2} \in I\left(\mathcal{W}_{\sigma}(\mathcal{L})\right)$ and assume $\lambda_{1} \leq \lambda_{2}$ on $\mathcal{W}_{\sigma}(\mathcal{L})$. Then $\lambda_{1}=\mu_{1}^{\prime}$ and $\lambda_{2}=\mu_{2}^{\prime}$ where $\mu_{1}, \mu_{2} \in I(\mathcal{L})$ and $\mu_{1}^{\prime} \leq \mu_{2}^{\prime}\left(\mathcal{W}_{\sigma}(\mathcal{L})\right)$ i.e $\mu_{1} \leq \mu_{2}(\mathcal{L})$. Now $S\left(\mu_{2}^{\prime}\right) \subset S\left(\mu_{1}^{\prime}\right)$. If $\lambda \in S\left(\mu_{1}^{\prime}\right)$, then clearly $\lambda \in I_{R}^{o}(\mathcal{L})$ and $\mu_{1} \leq \lambda(\mathcal{L})$ Hence by the assumption $\mu_{2} \leq \lambda(\mathcal{L})$ which implies $\lambda \in S\left(\mu_{2}^{\prime}\right)$

b) Suppose $I_{R}^{\sigma}(\mathcal{L}), \tau \mathcal{W}_{\sigma}(\mathcal{L})$ is $T_{2}$ which implies that $\mathcal{W}_{\sigma}(\mathcal{L})$ is $T_{2}$, and let $\mu, \nu_{1}, \nu_{2}$ as above. Then $\mu^{\prime} \leq \nu_{1}^{\prime}$ on $\mathcal{W}_{\sigma}(\mathcal{L})$ where $\mu^{\prime} \in I\left(\mathcal{W}_{\sigma}(\mathcal{L})\right)$ and $\nu_{1}^{\prime} \in I_{R}^{\sigma}\left(\mathcal{W}_{\sigma}(\mathcal{L})\right)$, which implies $\nu_{1} \in S\left(\nu_{1}^{\prime}\right) \subset S\left(\mu^{\prime}\right)$. Also $\mu^{\prime} \leq \nu_{2}^{\prime}$ on $\mathcal{W}_{\sigma}(\mathcal{L})$ where $\nu_{2}^{\prime} \in I_{R}^{\sigma}\left(W_{\sigma}(\mathcal{L})\right)$, which implies $\nu_{2} \in S\left(\nu_{2}^{\prime}\right) \subset S\left(\mu^{\prime}\right) \quad$ Recall that $\mathcal{L}$ is $T_{2}$ iff for each $\mu \in I(\mathcal{L}), S(\mu)=\emptyset$ or a singleton, hence since $\dot{\mathcal{W}}_{\sigma}(\mathcal{L})$ if $T_{2}$ it follows $\nu_{1}=\nu_{2}$. Conversely, assume that for $\mu \in I(\mathcal{L})$ and $\nu_{1}, \nu_{2} \in I_{R}^{\sigma}(\mathcal{L})$, if $\mu \leq \nu_{1}(\mathcal{L})$ and $\mu \leq \nu_{2}(\mathcal{L})$ then $\nu_{1}=\nu_{2}$. Suppose $S\left(\mu^{\prime}\right) \neq \emptyset$, where $\mu \in I(\mathcal{L}), \lambda \in I\left(\mathcal{W}_{\sigma}(\mathcal{L})\right)$ and $\lambda=\mu^{\prime} \quad$ If $\nu_{1}, \nu_{2} \in S\left(\mu^{\prime}\right)$ then $\mu \leq \nu_{1}(\mathcal{L})$ and $\mu \leq \nu_{2}(\mathcal{L})$ i e. $\nu_{1}=\nu_{2}$. Therefore $\mathcal{W}_{\sigma}(\mathcal{L})$ is $T_{2}$ and thus $\tau \mathcal{W}_{\sigma}(\mathcal{L})$ is $T_{2}$.

We now show that the assumption of regularity for $\mathcal{V}_{\sigma}(\mathcal{L})$ in $I_{\sigma}(\mathcal{L})$ is very strong.

THEOREM 3.2 Consider $I_{\sigma}(\mathcal{L})$ and $\mathcal{V}_{\sigma}(\mathcal{L})$. $\mathcal{V}_{\sigma}(\mathcal{L})$ is regular iff $I_{\sigma}(\mathcal{L})=I_{R}^{\sigma}(\mathcal{L})$

PROOF. Suppose $I_{\sigma}(\mathcal{L})=I_{R}^{\sigma}(\mathcal{L})$ Then $\mathcal{V}_{\sigma}(\mathcal{L})=\mathcal{W}_{\sigma}(\mathcal{L}) \quad$ Now let $\mu_{1}, \mu_{2} \in I(\mathcal{L}), \nu \in I_{\sigma}(\mathcal{L})$ and $\mu_{1} \leq \mu_{2}(\mathcal{L}), \mu_{1} \leq \nu(\mathcal{L})$. Then, since $I_{\sigma}(\mathcal{L})=I_{R}^{\sigma}(\mathcal{L}), \mu_{1} \in I_{R}^{\sigma}(\mathcal{L})$ so $\mu_{1}=\mu_{2}$ and $\mu_{1}=\nu$ Conversely, suppose $\mathcal{V}_{\sigma}(\mathcal{L})$ is regular and let $\mu \in I_{\sigma}(\mathcal{L})$; there exists $\nu \in I_{R}(\mathcal{L})$ such that $\mu \leq \nu(\mathcal{L})$ i.e $\mu^{\prime} \leq \nu^{\prime}\left(\mathcal{V}_{\sigma}(\mathcal{L})\right)$, where $\mu^{\prime}, \nu^{\prime} \in I_{\sigma}\left(\mathcal{V}_{\sigma}(\mathcal{L})\right)$ But $S\left(\mu^{\prime}\right)=S\left(\nu^{\prime}\right)$ since $\mathcal{V}_{\sigma}(\mathcal{L})$ is regular. Hence $\mu \in S\left(\nu^{\prime}\right)$ i.e. $\nu \leq \mu(\mathcal{L})$. It follows $\mu=\nu$ and then $\mu \in I_{R}^{o}(\mathcal{L})$.

\section{ON NORMAL, SLIGHTLY NORMAL, MILDLY NORMAL AND LINDELÖF LATTICES}

In this section we wish to consider normality and related questions as well as Lindelöf properties concerning the lattices $\mathcal{W}_{\sigma}(\mathcal{L})$ in $I_{R}^{\sigma}(\mathcal{L})$ and $\mathcal{V}_{\sigma}(\mathcal{L})$ in $I_{\sigma}(\mathcal{L})$. Related details on normality can be found in [4], [7] and [9]. We recall some definitions.

\section{DEFINITION 4.1}

a) $\mathcal{L}$ is slightly normal if for all $\mu \in I_{\sigma}\left(\mathcal{L}^{\prime}\right)$, there exists a unique $\nu \in I_{R}(\mathcal{L})$ such that $\mu \leq \nu(\mathcal{L})$

b) $\mathcal{L}$ is mildly normal if for all $\mu \in I_{\sigma}(\mathcal{L})$, there exists a unique $\nu \in I_{R}(\mathcal{L})$ such that $\mu \leq \nu(\mathcal{L})$

c) $\mathcal{L}$ is almost countably compact if $\mu \in I_{R}\left(\mathcal{L}^{\prime}\right)$ implies $\mu \in I_{\sigma}(\mathcal{L})$.

THEOREM 4.1 Suppose $\mathcal{L}$ is disjunctive. Then

a) Consider $I_{R}^{\sigma}(\mathcal{L})$ and $\mathcal{W}_{\sigma}(\mathcal{L})$ and suppose $\mathcal{L}$ is Lindelöf and satisfies the condition: for all $\mu_{1}, \mu_{2} \in I(\mathcal{L})$ and $\nu \in I_{R}^{\sigma}(\mathcal{L})$, if $\mu_{1} \leq \mu_{2}(\mathcal{L})$ and $\mu_{1} \leq \nu(\mathcal{L})$, then $\mu_{2} \leq \nu(\mathcal{L})$ Then $\mathcal{W}_{\sigma}(\mathcal{L})$ is slightly and mildly normal.

b) If $\mathcal{L}$ is complement generated then $\mathcal{W}_{\sigma}(\mathcal{L})$ is slightly normal.

c) If $\mathcal{L}$ is almost countably compact and mildly normal then $\mathcal{W}_{\sigma}(\mathcal{L})$ is normal.

PROOF. a) $\mathcal{L}$ is disjunctive and Lindelöf implies $\mathcal{W}_{\sigma}(\mathcal{L})$ Lindelöf. Also, by Theorem 3.1 it follows that $\mathcal{W}_{\sigma}(\mathcal{L})$ is regular. Then $\mathcal{W}_{\sigma}(\mathcal{L})$ is slightly and mildly normal (see [4]) 
b) $\mathcal{L}$ is complement generated implies $L=\bigcap_{1}^{\infty} L_{n}^{\prime}, L$ and $L_{n} \in \mathcal{L}$, all $n$

$$
W_{\sigma}(L)=W_{\sigma}\left(\bigcap_{1}^{\infty} L_{n}^{\prime}\right)=\bigcap_{1}^{\infty} W_{\sigma}\left(L_{n}^{\prime}\right)=\bigcap_{1}^{\infty}\left[W_{\sigma}\left(L_{n}\right)\right]^{\prime} .
$$

Hence $\mathcal{W}_{\sigma}(\mathcal{L})$ complement generated which implies $\mathcal{W}_{\sigma}(\mathcal{L})$ slightly normal (see [4])

c) By the assumption, for any $\mu \in I_{R}\left(\mathcal{L}^{\prime}\right)$ it follows $\mu \in I_{\sigma}(\mathcal{L})$ and then there exists a unique $\nu \in I_{R}(\mathcal{L})$ such that $\mu \leq \nu(\mathcal{L})$. Let $\mu \in I(\mathcal{L})$ such that $\mu \leq \lambda\left(L^{\prime}\right)$ with $\mu \in I_{R}\left(\mathcal{L}^{\prime}\right)$ Also $\lambda \in I_{\sigma}(\mathcal{L})$ and $\lambda \leq \mu \leq \nu$, on $\mathcal{L}$ with $\nu_{1} \in I_{R}(\mathcal{L})$, unique. Therefore if $\mu \leq \nu_{2}(\mathcal{L})$ with $\nu_{2} \in I_{R}(\mathcal{L})$ then $\lambda \leq \mu \leq \nu_{2}(\mathcal{L})$, and so $\nu_{1}=\nu_{2}$. Hence $\mathcal{L}$ is normal and also $\mathcal{W}_{\sigma}(\mathcal{L})$ is normal.

REMARK. Consider $I_{\sigma}(\mathcal{L})$ and $\mathcal{V}_{\sigma}(\mathcal{L})$ with $\mathcal{L}$ Lindelöf. If for all $\mu_{1}, \mu_{2} \in I(\mathcal{L})$ and $\nu \in I_{\sigma}(\mathcal{L})$ such that if $\mu_{1} \leq \mu_{2}(\mathcal{L})$ and $\mu_{1} \leq \nu(\mathcal{L})$ it follows that $\mu_{2} \leq \nu(\mathcal{L})$, then $\mathcal{V}_{\sigma}(\mathcal{L})$ is slightly and mildly normal.

PROOF. Similar to a) of Theorem 4.1.

We next consider the following condition:

$$
\text { For any } \Pi \in \pi_{\sigma}(\mathcal{L}) \text {, there exists } \nu \in I_{\sigma}(\mathcal{L}) \text { such that } \Pi \leq \nu(\mathcal{L})
$$

\section{THEOREM 4.2.}

a) If condition (1) is satisfies and if $\mathcal{L}$ is prime complete then $\mathcal{L}$ is Lindelöf.

b) If $\mathcal{L}$ is Lindelöf then condition (1) holds.

c) $\mathcal{L}$ satisfies condition $(1)$ iff $I_{\sigma}(\mathcal{L}), \tau \mathcal{V}_{\sigma}(\mathcal{L})$ is Lindelöf.

PROOF. a) Let $\Pi \in \pi_{\sigma}(\mathcal{L})$ be an $\mathcal{L}$-filter with the countable intersection property By condition (1) there exists $\nu \in I_{\sigma}(\mathcal{L})$ and $\Pi \leq \nu(\mathcal{L})$. $\mathcal{L}$ prime complete implies $S(\nu) \neq \emptyset$ and then $S(\Pi) \neq \emptyset$

b) Let $\Pi \in \pi_{\sigma}(\mathcal{L})$. Since $\mathcal{L}$ is Lindelöf, $S(\Pi) \neq \emptyset$ and therefore there exists $x \in X$ such that $x \in S(\Pi)$ Then $\Pi \leq \mu_{x}(\mathcal{L})$ and $\mu_{x} \in I_{\sigma}(\mathcal{L})$.

c) Suppose that $\mathcal{L}$ satisfies condition (1). Let $\Pi^{\prime} \in \pi_{\sigma}\left(\mathcal{V}_{\sigma}(\mathcal{L})\right)$ and define $\pi(L)=\Pi^{\prime}\left(V_{\sigma}(L)\right)$, $L \in \mathcal{L}$. If $L_{n} \downarrow \emptyset, L_{n} \in \mathcal{L}$ then $V_{\sigma}\left(L_{n}\right) \downarrow \emptyset$ and $\Pi\left(L_{n}\right)=\Pi^{\prime}\left(V_{\sigma}\left(L_{n}\right)\right) \rightarrow 0$, i.e. $\Pi \in \pi_{\sigma}(\mathcal{L})$ By condition (1), there exists $\nu \in I_{\sigma}(\mathcal{L})$ such that $\Pi \leq \nu(\mathcal{L})$. Hence $\nu^{\prime} \in I_{\sigma}\left(\mathcal{V}_{\sigma}(\mathcal{L})\right)$ and $\Pi^{\prime} \leq \nu^{\prime}$ on $\mathcal{V}_{\sigma}(\mathcal{L})$, where $\nu^{\prime}\left(V_{\sigma}(L)\right)=\nu(L), L \in \mathcal{L}$. Therefore $\mathcal{V}_{\sigma}(\mathcal{L})$ satisfies condition (1). Next, we show that $\mathcal{V}_{\sigma}(\mathcal{L})$ is prime complete. For this, let $S\left(\nu^{\prime}\right)=\bigcap_{\alpha \in A}\left\{V_{\sigma}\left(L_{\alpha}\right) / \nu^{\prime}\left(V_{\sigma}\left(L_{\alpha}\right)\right)=1, L_{\alpha} \in \mathcal{L}\right\}$. But $\nu^{\prime}\left(V_{\sigma}(L)\right)=1$ iff $\nu(L)=1$, iff $\nu \in V_{\sigma}(L)=\left\{\mu \in I_{\sigma}(\mathcal{L}) / \mu(L)=1, L \in \mathcal{L}\right\}$. Hence $V_{\sigma}(L) \neq \emptyset$ which implies $S\left(\nu^{\prime}\right) \neq \emptyset$ Now, $\mathcal{V}_{\sigma}(\mathcal{L})$ satisfies condition (1) and prime complete implies $\mathcal{V}_{\sigma}(\mathcal{L})$ Lindelof and then $\tau \mathcal{V}_{\sigma}(\mathcal{L})$ is Lindelöf. Conversely, let $\left(I_{\sigma}(\mathcal{L}), \tau \mathcal{V}_{\sigma}(\mathcal{L})\right)$ be Lindelöf Let $\Pi \in \pi_{\sigma}(\mathcal{L})$ and define $\Pi^{\prime}\left(V_{\sigma}(L)\right)=\Pi(L), L \in \mathcal{L}$. Then $V_{\sigma}\left(L_{n}\right) \downarrow \emptyset$ implies $L_{n} \downarrow \emptyset$ and $\Pi\left(L_{n}\right)=\Pi^{\prime}\left(V_{\sigma}\left(L_{n}\right)\right) \rightarrow 0$, hence $\Pi^{\prime} \in \pi_{\sigma}\left(\mathcal{V}_{\sigma}(\mathcal{L})\right) . \quad \tau \mathcal{V}_{\sigma}(\mathcal{L})$ Lindelöf implies $\mathcal{V}_{\sigma}(\mathcal{L})$ Lindelöf and then $\mathcal{V}_{\sigma}(\mathcal{L})$ satisfies condition (1), hence there exists $\nu^{\prime} \in I_{\sigma}\left(\mathcal{V}_{\sigma}(\mathcal{L})\right)$ such that $\Pi^{\prime} \leq \nu^{\prime}\left(\mathcal{V}_{\sigma}(\mathcal{L})\right)$, where $\nu^{\prime}\left(V_{\sigma}(L)\right)=\nu(L), L \in \mathcal{L} \quad \Pi(L)=1$ implies $\Pi^{\prime}\left(V_{\sigma}(L)\right)=1$ and then $\nu^{\prime}\left(V_{\sigma}(L)\right)=1$ i.e. $\nu(L)=1, L \in \mathcal{L}$. Hence $\Pi \leq \nu(\mathcal{L})$.

REMARK. If $\mathcal{L}$ is disjunctive and if for each $\Pi \in \pi_{\sigma}(\mathcal{L})$, there exists a $\nu \in I_{R}^{o}(\mathcal{L})$ such that $\Pi \leq \nu(\mathcal{L})$ then $I_{R}^{\sigma}(\mathcal{L}), \tau \mathcal{W}_{\sigma}(\mathcal{L})$ is Lindelöf. This result is known and appears e.g. in [8].

\section{ON PRIME COMPLETE AND COUNTABLY COMPACT LATTICES}

In this section we investigate the equivalence and consequences of stronger lattice completeness assumption.

THEOREM 5.1 Let $\mathcal{L}$ be a disjunctive lattice $\mathcal{W}_{\sigma}(\mathcal{L})$ is prime complete iff for $\mu \in I_{\sigma}(\mathcal{L})$ there exists $\nu \in I_{R}^{o}(\mathcal{L})$ such that $\mu \leq \nu(\mathcal{L})$.

PROOF. Let $\mu \in I_{\sigma}(\mathcal{L})$ and the associated $\mu^{\prime} \in I_{\sigma}\left(\mathcal{W}_{\sigma}(\mathcal{L})\right)$ defined by $\mu^{\prime}\left(W_{\sigma}(L)\right)=\mu(L), L \in \mathcal{L}$ If $\mathcal{W}_{\sigma}(\mathcal{L})$ is prime complete, $S\left(\mu^{\prime}\right) \neq \emptyset$ and then there exists $\nu \in S\left(\mu^{\prime}\right), \nu \in I_{R}^{\sigma}(\mathcal{L})$ and it follows 
that $\mu \leq \nu(\mathcal{L})$. Conversely, let $\mu^{\prime} \in I_{\sigma}\left(\mathcal{W}_{\sigma}(\mathcal{L})\right)$ and consider the associated $\mu \in I_{\sigma}(\mathcal{L})$ such that $\mu^{\prime}\left(W_{\sigma}(L)\right)=\mu(L)$. For $\mu \in I_{\sigma}(\mathcal{L})$, there exists $\nu \in I_{R}^{o}(\mathcal{L})$ such that $\mu \leq \nu(\mathcal{L})$. Therefore $\nu^{\prime} \in I_{R}^{\sigma}\left(\mathcal{W}_{\sigma}(\mathcal{L})\right)$ and $\mu^{\prime} \leq \nu^{\prime}\left(\mathcal{W}_{\sigma}(\mathcal{L})\right)$ which implies $S\left(\nu^{\prime}\right) \subset S\left(\mu^{\prime}\right)$ and since $\mathcal{W}_{\sigma}(\mathcal{L})$ is replete, $S\left(\nu^{\prime}\right) \neq \emptyset$

\section{THEOREM 5.2}

a) Let $\mathcal{L}$ be disjunctive, almost countably compact and mildly normal and let $\mathcal{W}_{\sigma}(\mathcal{L})$ be prime complete. Then $\mathcal{L}$ is countably compact.

b) Let $\mathcal{L}$ be disjunctive, regular, Lindelöf, almost countably compact and let $\mathcal{W}_{\sigma}(\mathcal{L})$ be prime complete. Then $\mathcal{L}$ is countably compact.

PROOF. a) Must show that $I_{R}(\mathcal{L})=I_{R}^{\sigma}(\mathcal{L})$. Let $\mu \in I_{R}(\mathcal{L})$; we have $\mu \leq \nu\left(\mathcal{L}^{\prime}\right)$ where $\nu \in I_{R}\left(\mathcal{L}^{\prime}\right)$. Since $\mathcal{L}$ is almost countably compact we have $\nu \leq \mu(\mathcal{L})$ with $\mu \in I_{R}(\mathcal{L})$ and $\nu \in I_{\sigma}(\mathcal{L})$ But $\mathcal{W}_{\sigma}(\mathcal{L})$ is prime complete and by Theorem 5.1 there exists $\rho \in I_{R}^{o}(\mathcal{L})$ such that $\nu \leq \rho(\mathcal{L}) \quad \mathcal{L}$ almost countably compact and mildly normal implies $\mathcal{L}$ normal (see [4]). By the normality of $\mathcal{L}$ the $\mathcal{L}$-regular measure $\mu$ such that $\nu \leq \mu$ must be unique, hence $\mu=\rho \in I_{R}^{o}(\mathcal{L})$.

b) $\mathcal{L}$ regular and Lindelof implies $\mathcal{L}$ mildly normal and by the above result, it follows that $\mathcal{L}$ is countably compact.

THEOREM 5.3 Suppose $I_{\sigma}(\mathcal{L}), \mathcal{V}_{\sigma}(\mathcal{L})$ is $T_{1}$ and $\mathcal{L}$ disjunctive and $\mathcal{W}_{\sigma}(\mathcal{L})$ prime complete Then $I_{\sigma}(\mathcal{L})=I_{R}^{\sigma}(\mathcal{L})$

PROOF. Since $I_{\sigma}(\mathcal{L}), \mathcal{V}_{\sigma}(\mathcal{L})$ is $T_{1}$, given $\mu_{1} \neq \mu_{2}$ with $\mu_{1}, \mu_{2} \in I_{\sigma}(\mathcal{L})$, there exist $L_{1}, L_{2} \in \mathcal{L}$ such that $\mu_{1} \in V_{\sigma}\left(L_{1}^{\prime}\right), \mu_{2} \notin V_{\sigma}\left(L_{1}^{\prime}\right)$ and $\mu_{2} \in V_{\sigma}\left(L_{2}^{\prime}\right), \mu_{1} \notin V_{\sigma}\left(L_{2}^{\prime}\right)$. Therefore $\mu_{1}\left(L_{1}^{\prime}\right)=1, \mu_{2}\left(L_{1}^{\prime}\right)=0$ or $\mu_{1}\left(L_{1}\right)=0, \mu_{2}\left(L_{1}\right)=1$ and $\mu_{2}\left(L_{2}^{\prime}\right)=1, \mu_{1}\left(L_{2}^{\prime}\right)=0$ or $\mu_{2}\left(L_{2}\right)=0, \mu_{1}\left(L_{2}\right)=1$. Since $\mathcal{W}_{\sigma}(\mathcal{L})$ prime complete, given $\mu \in I_{\sigma}(\mathcal{L})$ there exists $\nu \in I_{R}^{\sigma}(\mathcal{L})$ with $\mu \leq \nu(\mathcal{L})$. If $\mu \neq \nu$, by above there exists $L \in \mathcal{L}$ such that $\nu(L)=0$ and $\mu(L)=1$

This is a contradiction, hence $\mu=\nu$, and $I_{\sigma}(\mathcal{L})=I_{R}^{\sigma}(\mathcal{L})$.

REMARK. The converse of Theorem 5.3 is true i.e. if $I_{\sigma}(\mathcal{L})=I_{R}^{\sigma}(\mathcal{L})$ then $\mathcal{V}_{\sigma}(\mathcal{L})=\mathcal{W}_{\sigma}(\mathcal{L})$ is $T_{1}$ and since $\mathcal{V}_{\sigma}(\mathcal{L})$ is prime complete, then $\mathcal{W}_{\sigma}(\mathcal{L})$ is prime complete.

DEFINITION 5.1 Let $\mu \in I(\mathcal{L}), E \subset X$ and define

$$
\mu^{\prime}(E)=\inf \left\{\sum_{\imath=1}^{n} \mu\left(L_{\imath}^{\prime}\right), E \subset \bigcup_{\imath=1}^{n} L_{\imath}^{\prime}, L_{\imath} \in \mathcal{L}\right\}=\inf \left\{\mu\left(L^{\prime}\right), E \subset L^{\prime}, L \in \mathcal{L}\right\} .
$$

DEFINITION 5.2 Let $\mu \in I_{\sigma}(\mathcal{L}), E \subset X$ and define

$$
\mu^{\prime \prime}(E)=\inf \left\{\sum_{\imath=1}^{\infty} \mu\left(L_{\imath}^{\prime}\right), E \subset \bigcup_{\imath=1}^{\infty} L_{\imath}^{\prime}, L_{\imath} \in \mathcal{L}\right\} .
$$

Clearly, $\mu^{\prime}$ is a finitely subadditive outer measure and $\mu^{\prime \prime}$ is an outer measure (see [7]). Let $\mathfrak{I}_{\mu^{\prime \prime}}$ be the set of $\mu^{\prime \prime}$-measurable sets, where $E$ is measurable with respect to $\mu^{\prime \prime}$ if for any $A \subset X$, $\mu^{\prime \prime}(A)=\mu^{\prime \prime}(A \cap E)+\mu^{\prime \prime}\left(A \cap E^{\prime}\right)$.

We give now a condition when for a given $\mu \in I_{\sigma}(\mathcal{L})$ there exists a $\nu \in I_{R}^{o}(\mathcal{L})$ such that $\mu \leq \nu(\mathcal{L})$ i e. the condition of Theorem 5.1.

THEOREM 5.4 Let $\mu \in I_{\sigma}(\mathcal{L})$. Suppose $\mathcal{L} \subset \mathcal{L}_{\mu^{\prime \prime}}$ and $\mathcal{L}$ semiseparates $\delta(\mathcal{L}) \quad$ Then $\mu \leq \mu^{\prime \prime}(\mathcal{L})$ and $\left.\mu^{\prime \prime}\right|_{\mathcal{A}(\mathcal{L})} \in I_{R}^{\sigma}(\mathcal{L})$.

PROOF. Let $\mu \in I_{\sigma}(\mathcal{L})$. Then we have $\mu \leq \mu^{\prime \prime}(\mathcal{L})$ and $\mathcal{L} \subset \mathcal{L}_{\mu^{\prime \prime}}$ which is closed under complement and countable unions (see [7]). Therefore $\mathcal{A}(\mathcal{L}) \subset \mathfrak{I}_{\mu^{\prime \prime}} .\left.\quad \mu^{\prime \prime}\right|_{\mathcal{A}(\mathcal{L})}$ is then a measure on $\mathcal{A}(\mathcal{L}) \quad \mu^{\prime \prime}$ countably additive implies $\left.\mu^{\prime \prime}\right|_{\mathcal{A}(\mathcal{L})} \in I^{\sigma}(\mathcal{L})$ To show that $\left.\mu^{\prime \prime}\right|_{\mathcal{A}(\mathcal{L})} \in I_{R}(\mathcal{L})$, assume $\mu^{\prime \prime}\left(A^{\prime}\right)=1$, $A \in \mathcal{L}$. Then there exist $\left\{L_{n}\right\}, L_{n} \in \mathcal{L}$ such that $A^{\prime} \supseteq \bigcap_{n} L_{n}$ and $\mu\left(L_{n}\right)=1$ for all $n$ (For if $A \supset \bigcap L_{n}, \mu\left(L_{n}\right)=1$ then $A^{\prime} \subset \bigcup L_{n}^{\prime}, \mu\left(L_{n}^{\prime}\right)=0$, contradiction.) But $\bigcap_{n} L_{n} \in \delta(\mathcal{L})$ and $A \bigcap$ 
$\left(\bigcap_{n} L_{n}\right)=\emptyset$. Hence by semiseparation there exists $\tilde{L} \in \mathcal{L}$ such that $A \cap \tilde{L}=\emptyset$ (or $\tilde{A} \subset A^{\prime}$ ) and $\bigcap_{n}$ $L_{n} \subset \tilde{L} \quad$ May assume $L_{n} \downarrow$ and then $\mu^{\prime \prime}\left(\bigcap L_{n}\right)=1$. We then have $\bigcap_{n} L_{n} \subset \tilde{L} \subset A^{\prime}$ which implies $\mu^{\prime \prime}(L)=1$ i.e. $\left.\mu^{\prime \prime}\right|_{\mathcal{A}(\mathcal{L})} \in I_{R}(\mathcal{L})$

THEOREM 5.5 Let $\mathcal{L}$ be a disjunctive lattice and assume that $\mathcal{L} \subset \bigcap_{\mu \in I_{\sigma}(\mathcal{L})} \mathfrak{I}_{\mu^{\prime \prime}}$ and that $\mathcal{L}$ semiseparates $\delta(\mathcal{L})$ Then $\mathcal{W}_{\sigma}(\mathcal{L})$ is prime complete.

PROOF. $\mathcal{W}_{\sigma}(\mathcal{L})$ is prime complete iff for any $\mu \in I_{\sigma}(\mathcal{L})$, there exists $\nu \in I_{R}^{\sigma}(\mathcal{L})$ such that $\mu \leq \nu(\mathcal{L})$, by Theorem 51 . Now use Theorem 54 with $\nu=\left.\mu^{\prime \prime}\right|_{\mathcal{A}(\mathcal{L})}$.

\section{STRONGLY $\sigma$-SMOOTH MEASURES}

Here we consider another Wallman space and analyze the relevant lattice in detail.

DEFINITION 6.1 A measure $\mu \in I(\mathcal{L})$ is strongly $\sigma$-smooth on $\mathcal{L}$ iff for any sequence $\left\{L_{n}\right\}$, $L_{n} \in \mathcal{L}, L_{n} \downarrow$, if $\bigcap_{n} L_{n} \in \mathcal{L}$ then $\mu\left(\bigcap L_{n}\right)=\inf \mu\left(L_{n}\right)=\lim _{n \rightarrow \infty} \mu\left(L_{n}\right)$. We denote $J(\mathcal{L})$ the set of strongly $\sigma$-smooth nontrivial zero-one valued measures on $\mathcal{L}$.

DEFINITION 6.2 The lattice $\mathcal{L}$ is weakly prime complete if for $\mu \in J(\mathcal{L}), S(\mu) \neq \emptyset$.

Now define the following condition:

$$
\text { For any } \Pi \in \pi_{\sigma}(\mathcal{L}) \text { there exists } \nu \in J(\mathcal{L}) \text { such that } \Pi \leq \nu(\mathcal{L}) \text {. }
$$

We summarize a few facts on $\sigma$-smoothness that will be used throughout this section for the reader's convenience (see [6])

a) $I^{\sigma}(\mathcal{L}) \subset J(\mathcal{L}) \subset I_{\sigma}(\mathcal{L})$.

b) $\mathcal{L}$ normal and complement generated implies $J(\mathcal{L}) \subset I_{R}^{\sigma}(\mathcal{L})$.

c) $\mu \in I_{\sigma}(\mathcal{L})$ and $\mu^{\prime}=\mu^{\prime \prime}(\mathcal{L})$ implies $\mu \in J(\mathcal{L})$.

d) Since $\mu \in I_{R}^{\sigma}(\mathcal{L})$ implies $\mu^{\prime}=\mu^{\prime \prime}\left(\mathcal{L}^{\prime}\right)$, it follows that $\mu \in J(\mathcal{L})$ and then $I_{R}^{o}(\mathcal{L}) \subset J(\mathcal{L})$.

Analogously to Theorem 4.2 we have

\section{THEOREM 6.1}

a) If condition (2) holds and if $\mathcal{L}$ is weakly prime complete then $\mathcal{L}$ is Lindelöf.

b) If $\mathcal{L}$ is Lindelöf then condition (2) holds.

PROOF. Omitted.

THEOREM 6.2 Define $\mathcal{V}_{J}(\mathcal{L})=\left\{V_{J}(L) / L \in \mathcal{L}\right\}$ where $V_{J}(L)=\{\mu \in J(\mathcal{L}) / \mu(L)=1, L \in \mathcal{L}\}$ Then $\mathcal{L}$ satisfies condition (2) iff $J(\mathcal{L}), \tau \mathcal{V}_{J}(\mathcal{L})$ is Lindelöf.

PROOF. Suppose $\mathcal{L}$ satisfies condition (2), we show that $\mathcal{V}_{J}(\mathcal{L})$ satisfies condition (2) For this, let $\Pi^{\prime} \in \pi_{\sigma}\left(\mathcal{V}_{J}(\mathcal{L})\right)$ and define $\Pi(L)=\Pi^{\prime}\left(V_{\jmath}(L)\right), L \in \mathcal{L} . \quad$ If $L_{n} \downarrow \emptyset, L_{n} \in \mathcal{L}$ then $V_{J}\left(L_{n}\right) \downarrow \emptyset$ and $\lim _{n} \Pi\left(L_{n}\right)=\lim _{n} \Pi^{\prime}\left(V_{J}\left(L_{n}\right)\right)=0$, hence $\Pi \in \pi_{\sigma}(\mathcal{L})$. By condition $(2)$ there exists $\nu \in J(\mathcal{L})$ such that $\Pi \leq \nu(\mathcal{L})$. Hence $\nu^{\prime} \in J\left(\mathcal{V}_{J}(\mathcal{L})\right)$ and $\Pi^{\prime} \leq \nu^{\prime}$ on $\mathcal{V}_{J}(\mathcal{L})$ where $\nu^{\prime}\left(V_{J}(L)\right)=\nu(L), L \in \mathcal{L}$. For $\Pi^{\prime} \in \pi_{\sigma}\left(\mathcal{V}_{J}(\mathcal{L})\right)$, there exists $\nu^{\prime} \in J\left(\mathcal{V}_{J}(\mathcal{L})\right)$ such that $\Pi^{\prime} \leq \nu^{\prime}$ on $\mathcal{V}_{J}(\mathcal{L})$.

Next we show that $\mathcal{V}_{J}(\mathcal{L})$ is weakly prime complete; let $S(\nu)=\bigcap_{\alpha \in A}\left\{V_{J}\left(L_{\alpha}\right) / \nu^{\prime}\left(V_{J}\left(L_{\alpha}\right)\right)=1, L_{\alpha} \in \mathcal{L}\right\}$. $\nu^{\prime}\left(V_{J}(L)\right)=1$ iff $\nu(L)=1$ iff $\nu \in V_{J}(L)=\{\mu \in J(\mathcal{L}) / \mu(L)=1, L \in \mathcal{L}\}$. Hence $V_{J}(L) \neq \emptyset$ implies $S\left(\nu^{\prime}\right) \neq \emptyset$. Therefore $\mathcal{V}_{J}(\mathcal{L})$ is Lindelöf, and then $\tau \mathcal{V}_{J}(\mathcal{L})$ is Lindelöf. Conversely, assume $\left(J(\mathcal{L}), \tau \mathcal{V}_{J}(\mathcal{L})\right)$ is Lindelöf and let $\Pi \in \pi_{\sigma}(\mathcal{L})$. Define $\Pi^{\prime}\left(V_{J}(L)\right)=\Pi(L), L \in \mathcal{L}$. Then $V_{J}\left(L_{n}\right) \downarrow \emptyset$ which implies $L_{n} \downarrow \emptyset$ and $\lim _{n} \Pi\left(L_{n}\right)=\lim _{n} \Pi^{\prime}\left(V_{J}\left(L_{n}\right)\right)=0$ i.e. $\Pi^{\prime} \in \pi_{\sigma}\left(\mathcal{V}_{J}(\mathcal{L})\right) . \tau \mathcal{V}_{J}(\mathcal{L})$ Lindelöf, then $\mathcal{V}_{J}(\mathcal{L})$ Lindelöf, then $\mathcal{V}_{J}(\mathcal{L})$ satisfies condition (2). Hence there exists $\nu^{\prime} \in J\left(\mathcal{V}_{J}(\mathcal{L})\right)$ such that $\Pi^{\prime} \leq \nu^{\prime}$ on $\mathcal{V}_{J}(\mathcal{L})$, where $\nu^{\prime}\left(V_{J}(L)\right)=\nu(L), L \in \mathcal{L}$. Therefore $\Pi \leq \nu(\mathcal{L})$.

Again, analogous to our previous work we have:

THEOREM 6.3 Consider $J(\mathcal{L})$ and $\mathcal{V}_{J}(\mathcal{L}) . \quad \mathcal{V}_{J}(\mathcal{L})$ is regular iff for all $\mu_{1}, \mu_{2} \in I(\mathcal{L})$ and $\nu \in J(\mathcal{L})$, if $\mu_{1} \leq \mu_{2}(\mathcal{L})$ and $\mu_{1} \leq \nu(\mathcal{L})$ then $\mu_{2} \leq \nu(\mathcal{L})$ 
PROOF. For $\mu_{1}, \mu_{2} \in I(\mathcal{L})$ we have $\mu_{1}^{\prime}, \mu_{2}^{\prime} \in I\left(\mathcal{W}_{\sigma}(\mathcal{L})\right)$ and then $\mu_{1}^{\prime}, \mu_{2}^{\prime} \in I\left(\mathcal{V}_{J}(\mathcal{L})\right)$, $\mu_{1}^{\prime}\left(V_{J}(L)\right)=\mu_{1}(L)$ and $\mu_{2}^{\prime}\left(V_{J}(L)\right)=\mu_{2}(L)$. If $\mathcal{V}_{J}(\mathcal{L})$ is regular then $S\left(\mu_{1}^{\prime}\right)=S\left(\mu_{2}^{\prime}\right)$, where $S\left(\mu_{1}^{\prime}\right)=\cap\left\{V_{J}(L) \in \mathcal{V}_{J}(\mathcal{L}) / \mu_{1}^{\prime}\left(V_{J}(L)=1, L \in \mathcal{L}\right\} \quad\right.$ Let $\nu \in J(\mathcal{L}) ; \nu^{\prime} \in J\left(\mathcal{V}_{J}(\mathcal{L})\right)$ and $\mu_{1}^{\prime} \leq \nu^{\prime}$ on $\mathcal{V}_{J}(\mathcal{L})$ Then $\nu \in S\left(\mu_{2}^{\prime}\right)$ i.e. $\mu_{2} \leq \nu(\mathcal{L})$. Conversely, suppose $\mu_{1}, \mu_{2} \in I(\mathcal{L})$ and $\nu \in J(\mathcal{L})$ such that if $\mu_{1} \leq \mu_{2}(\mathcal{L})$ and $\mu_{1} \leq \nu(\mathcal{L})$ then $\mu_{2} \leq \nu(\mathcal{L})$ Let $\lambda_{1}, \lambda_{2} \in I\left(\mathcal{V}_{J}(\mathcal{L})\right)$ and $\lambda_{1} \leq \lambda_{2}$ on $\mathcal{V}_{J}(\mathcal{L})$ Then $\lambda_{1}=\mu_{1}^{\prime}$ and $\lambda_{2}=\mu_{2}^{\prime}$ with $\mu_{1}, \mu_{2} \in I(\mathcal{L})$. Thus $\mu_{1}^{\prime} \leq \mu_{2}^{\prime}$ on $\mathcal{V}_{J}(\mathcal{L})$ which implies $\mu_{1} \leq \mu_{2}$ on $\mathcal{L}$, hence $S\left(\mu_{2}^{\prime}\right) \subset S\left(\mu_{1}^{\prime}\right)$. If $\lambda \in S\left(\mu_{1}^{\prime}\right)$ then clearly $\lambda \in J(\mathcal{L})$ and $\mu_{1} \leq \lambda(\mathcal{L})$. By the condition of the statement, $\mu_{2} \leq \lambda(\mathcal{L})$ and then $\lambda \in S\left(\mu_{2}^{\prime}\right)$. Hence $S\left(\mu_{2}^{\prime}\right)=S\left(\mu_{1}^{\prime}\right)$ and $\mathcal{V}_{J}(\mathcal{L})$ is regular

THEOREM 6.4 Consider $J(\mathcal{L}), \mathcal{V}_{J}(\mathcal{L})$. If $\mathcal{V}_{J}(\mathcal{L})$ is regular, then $J(\mathcal{L})=I_{R}^{\sigma}(\mathcal{L})$

PROOF. Let $\mu \in J(\mathcal{L})$. Then there exists $\nu \in I_{R}(\mathcal{L})$ such that $\mu \leq \nu(\mathcal{L})$, hence $\mu^{\prime} \leq \nu^{\prime}$ on $\mathcal{V}_{J}(\mathcal{L})$, where $\mu^{\prime} \in J\left(\mathcal{V}_{J}(\mathcal{L})\right)$ and $\nu^{\prime} \in I_{R}\left(\mathcal{V}_{J}(\mathcal{L})\right)$. $\mathcal{V}_{J}(\mathcal{L})$ regular implies $S\left(\mu^{\prime}\right)=S\left(\nu^{\prime}\right)$, therefore $\nu \leq \mu(\mathcal{L})$ Then $\mu=\nu(\mathcal{L})$ and since $\nu \in I_{R}(\mathcal{L}), J(\mathcal{L}) \subset I_{\sigma}(\mathcal{L})$ it follows that $\mu \in I_{R}(\mathcal{L}), I_{\sigma}(\mathcal{L})$ and then $\mu \in I_{R}^{\sigma}(\mathcal{L})$. Thus $J(\mathcal{L})=I_{R}^{\sigma}(\mathcal{L})$.

THEOREM 6.5 Consider $J(\mathcal{L})$ and $\mathcal{V}_{J}(\mathcal{L})$, with $\mathcal{L}$ Lindelof. If for all $\mu_{1}, \mu_{2} \in I(\mathcal{L})$ and $\nu \in J(\mathcal{L})$ such that if $\mu_{1} \leq \mu_{2}(\mathcal{L})$ and $\mu_{1} \leq \nu(\mathcal{L})$ then $\mu_{2} \leq \nu(\mathcal{L})$ it follows that $\mathcal{V}_{J}(\mathcal{L})$ is slightly and mildly normal

PROOF. By Theorem $6.3 \mathcal{V}_{J}(\mathcal{L})$ is regular. We show as in the Remark of Theorem 41 that $\mathcal{V}_{J}(\mathcal{L})$ is Lindelof and then, $\mathcal{V}_{J}(\mathcal{L})$ being regular and Lindelöf, it follows that it is also slightly and mildly normal

THEOREM 6.6 $\mathcal{V}_{J}(\mathcal{L})$ is prime complete iff for $\mu \in I_{\sigma}(\mathcal{L})$ there exists $\nu \in J(\mathcal{L})$ such that $\mu \leq \nu(\mathcal{L})$.

Let $\mu \in I_{\sigma}(\mathcal{L})$ such that there exists $\nu \in J(\mathcal{L})$ and $\mu \leq \nu(\mathcal{L})$. Consider the corresponding $\mu^{\prime} \in I_{\sigma}\left(\mathcal{V}_{J}(\mathcal{L})\right)$ and $\nu^{\prime} \in J\left(\mathcal{V}_{J}(\mathcal{L})\right)$ for which $\mu^{\prime} \leq \nu^{\prime}$ holds on $\mathcal{V}_{J}(\mathcal{L}) \quad \mathcal{V}_{J}(\mathcal{L})$ weakly prime complete implies $S\left(\nu^{\prime}\right) \neq \emptyset$ and since $\mu^{\prime} \leq \nu^{\prime}$ it follows $S\left(\mu^{\prime}\right) \neq \emptyset$, so that $\mathcal{V}_{J}(\mathcal{L})$ is prime complete. Conversely, suppose $\mathcal{V}_{J}(\mathcal{L})$ prime complete and let $\mu \in I_{\sigma}(\mathcal{L})$. Consider the corresponding $\mu^{\prime} \in I_{\sigma}\left(\mathcal{V}_{J}(\mathcal{L})\right.$ ), for which $S\left(\mu^{\prime}\right) \neq \emptyset$, so there exists $\nu \in S\left(\mu^{\prime}\right)$ and $\mu \leq \nu$ on $\mathcal{V}_{J}(\mathcal{L})$ Hence $\nu \in J(\mathcal{L})$.

We can now return to $I_{R}^{\sigma}(\mathcal{L})$ and the lattice $\mathcal{W}_{\sigma}(\mathcal{L})$, where $\mathcal{L}$ is disjunctive, and can ask when this lattice is weakly prime complete.

THEOREM 6.7 Let $\mathcal{L}$ be a disjunctive lattice and consider $I_{R}^{o}(\mathcal{L}) . \quad \mathcal{W}_{\sigma}(\mathcal{L})$ is weakly prime complete iff for $\mu \in J(\mathcal{L})$ there exists $\nu \in I_{R}^{o}(\mathcal{L})$ such that $\mu \leq \nu(\mathcal{L})$.

PROOF. Let $\mu \in J(\mathcal{L})$ and consider the associated $\mu^{\prime} \in J\left(\mathcal{W}_{\sigma}(\mathcal{L})\right)$ defined by $\mu^{\prime}\left(W_{\sigma}(L)\right)=\mu(L)$, $L \in \mathcal{L} \quad \mathcal{W}_{\sigma}(\mathcal{L})$ prime complete implies $S\left(\mu^{\prime}\right) \neq \emptyset$, and then there exists $\nu \in S\left(\mu^{\prime}\right)$ and $\mu \leq \nu(\mathcal{L})$, $\nu \in I_{R}^{\sigma}(\mathcal{L})$

Conversely, let $\mu^{\prime} \in J\left(\mathcal{W}_{\sigma}(\mathcal{L})\right)$ and consider the associated $\mu \in J(\mathcal{L})$ such that $\mu^{\prime}\left(W_{\sigma}(L)\right)=\mu(L)$, $L \in \mathcal{L}$. Assume now that there exists $\nu \in I_{R}^{\sigma}(\mathcal{L})$ such that $\mu \leq \nu(\mathcal{L})$. Then $\nu^{\prime} \in I_{R}^{\sigma}\left(\mathcal{W}_{\sigma}(\mathcal{L})\right)$ and $\mu^{\prime} \leq \nu^{\prime}$ on $\mathcal{W}_{\sigma}(\mathcal{L})$. Hence $S\left(\nu^{\prime}\right) \subset S\left(\mu^{\prime}\right)$ and since $\mathcal{W}_{\sigma}(\mathcal{L})$ is replete, $S\left(\nu^{\prime}\right) \neq \emptyset$.

We finally note that the conditions of Theorem 5.4 that $\mathcal{L} \in \mathfrak{I}_{\mu^{\prime \prime}}$ is very strong if $\mu \in J(\mathcal{L})$, namely.

THEOREM 6.8 Let $\mu \in J(\mathcal{L})$ and assume that $\mathcal{L} \subset \mathfrak{I}_{\mu^{\prime \prime}} \quad$ Then if there exists $\nu \in I_{R}^{\sigma}(\mathcal{L})$ such that $\mu \leq \nu(\mathcal{L})$ then $\mu=\nu$

PROOF. Suppose there exists $L \in \mathcal{L}$ such that $\mu(L)=0$ and $\nu(L)=1$ Now $\mu \leq \nu=\nu^{\prime \prime} \leq \mu^{\prime \prime}(\mathcal{L})$, therefore $\mu^{\prime \prime}(L)=1$, and since $\mathcal{L} \subset \mathfrak{I}_{\mu^{\prime \prime}}$ we get $\mu^{\prime \prime}\left(L^{\prime}\right)=0$. But $\mu \in J(\mathcal{L})$ iff $\mu=\mu^{\prime}=\mu^{\prime \prime}\left(\mathcal{L}^{\prime}\right)$ Therefore $\mu\left(L^{\prime}\right)=0$ which implies $\mu(X)=0$, contradiction. It follows that $\mu=\nu$

\section{REFERENCES}

[1] ALEXANDROFF, A.D., Additive set functions in abstract spaces, Mat. Sb. (N.S.) 8, 50 (1940), 307-348.

[2] BACHMAN, G. and STRATIGOS, P., Lattice repleteness and some of its applications to topology, J. Math. Anal. \& Appl., 99, 2 (1984), 472-492. 
[3] CONNELL, R., On certain Wallman spaces, Internat. J. Math. \& Math. Sci., 17, 2 (1994), 273 276.

[4] EID, G., On normal lattices and Wallman spaces, Internat. J. Math. \& Math. Scl., 13, 1 (1990), 3138.

[5] GRASSI, P., Outer measures and associated lattice properties, Internat. J. Math. \& Math. Scl., 16, 4 (1992), 687-694.

[6] HSU, P-S., Applications of outer measures to separation properties of lattices and regular or $\sigma$ smooth measures, Internat. J. Math. \& Math. Sci., 19, 2 (1996), 253-262.

[7] VLAD, C., Regular measures and normal lattices, Internat. J. Math. \& Math. Scl., 17, 3 (1994), 441-446.

[8] VLAD, C., Lattice separation and properties of Wallman type spaces, Annali di Matematica pura ed applicata, (IV), Vol. CLV (1991), 65-79.

[9] VLAD, C., On normal lattices and semiseparation of lattices, J. Indian Math. Soc., 56 (1991), 259273.

[10] WALLMAN, H., Lattices and topological spaces, Ann. of Math., 39 (1938), 112-126 


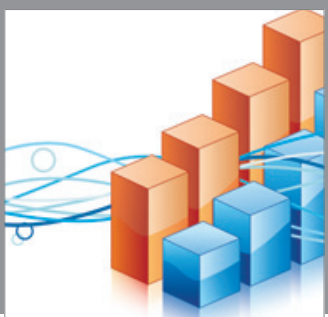

Advances in

Operations Research

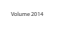

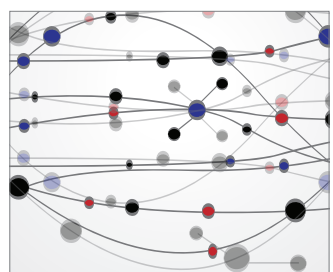

\section{The Scientific} World Journal
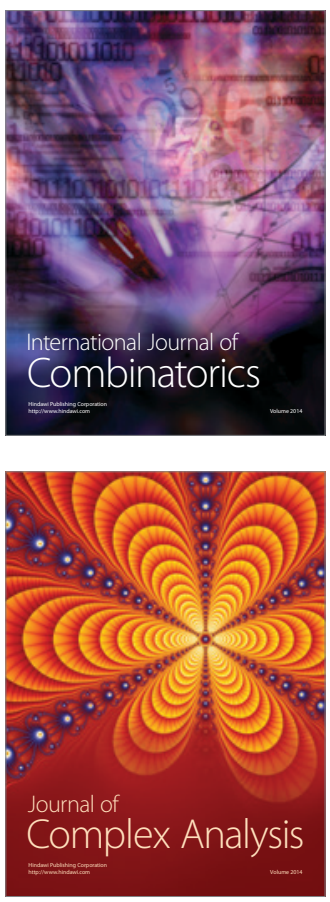

International Journal of

Mathematics and

Mathematical

Sciences
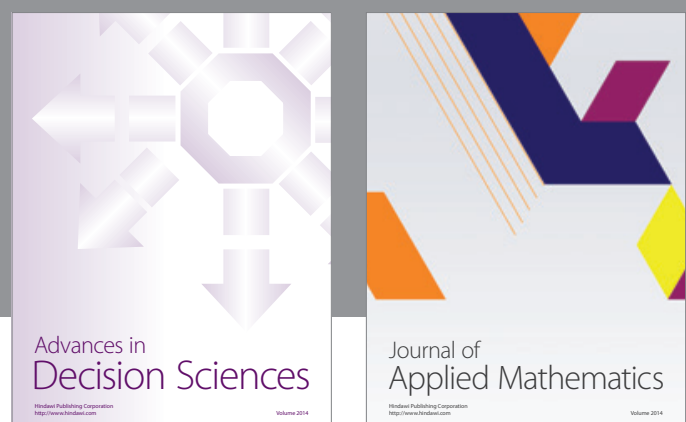

Journal of

Applied Mathematics
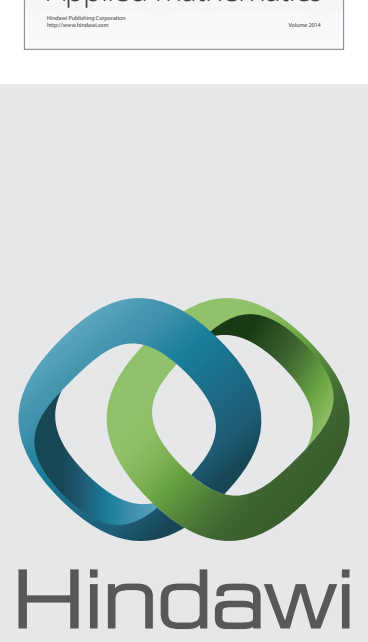

Submit your manuscripts at http://www.hindawi.com
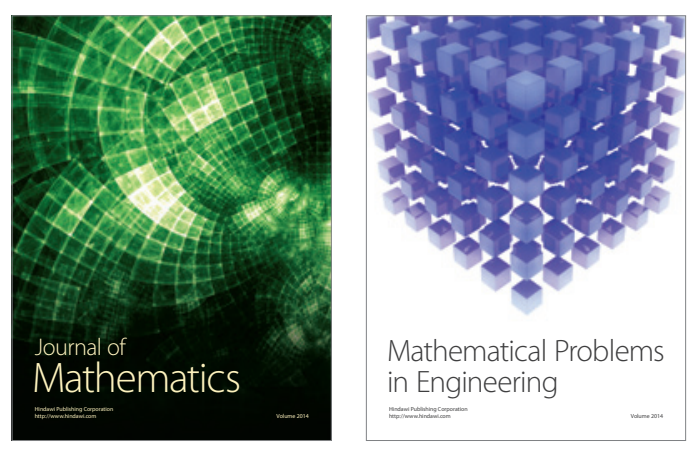

Mathematical Problems in Engineering
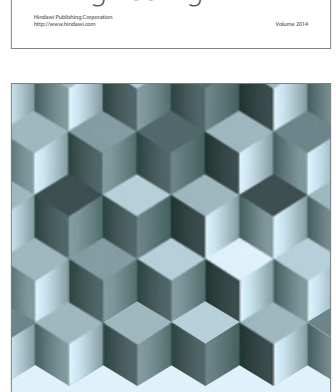

Journal of

Function Spaces
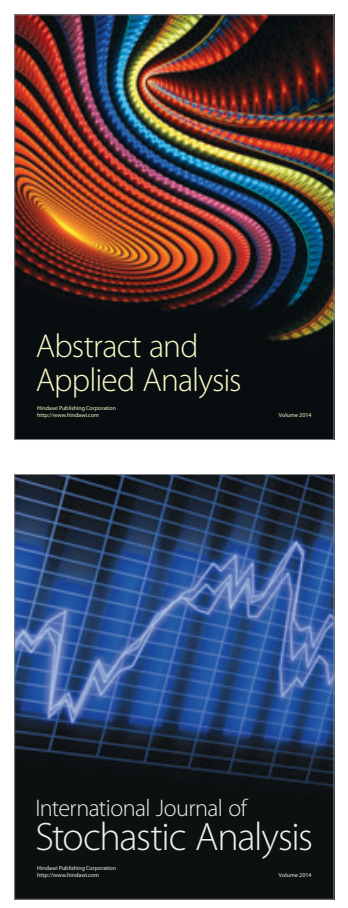

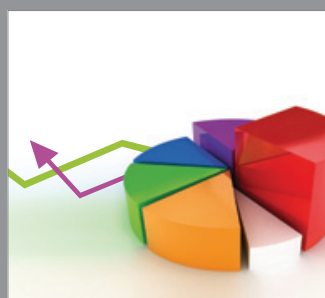

ournal of

Probability and Statistics

Promensencen
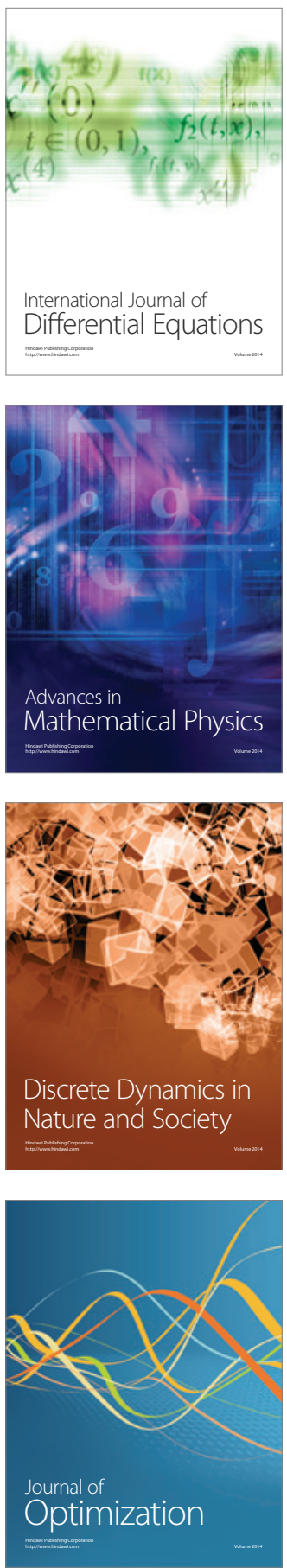